\title{
ACUTE LEUKAEMIC CONDITIONS IN CHILDHOOD
}

\author{
By Ursula Shelley, M.D., M.R.C.P. \\ Physician to Children's Department, Royal Free Hospital, London, Queen Elizabeth Hospital for Children, \\ Hackney, and Princess Louise Hospital for Children, Kensington
}

In any age group the leukaemic state is characterized by overgrowth of the leucopoietic tissues, in which an increased number of leucocytes, many of which are immature, rapidly develop. These young white cells are also found among the greatly increased total number of leucocytes in the circulating blood and it is on their presence and their type that the diagnosis depends. Occasionally, however, the total number of leucocytes may be normal or even decreased but in such cases the large proportion of those cells found in the blood stream will be young forms. This picture, of a normal or a reduced total number of white cells, most of which are immature, constitutes aleukaemic leukaemia. The diagnosis of leukaemia depends, therefore, on the nature and not on the number of circulating white cells. The symptoms and signs will depend on the site of origin of these abnormal cells, that is the bone marrow, the lymphatic glands or the reticuloendothelial system.

\section{Aetiology}

The causative factor in this disease entity is still unknown.

Two schools of thought are predominant. The first school holds that the tissue changes are due to neoplastic infiltration. As in other neoplastic conditions the manner of growth of the leukaemias is uncontrolled hyperplasia, together with production of immature cells and deposition of abnormal cells in all tissues of the body. The disease is invariably fatal. Experimental animals, which have been treated with agents such as tar, indol and other irritative fluids, known to produce malignant disease, have also been found to be suffering from acute leukaemia (Mallory, 1939). Against this malignant theory is the fact that the tissue changes are present, and may be demonstrated, all at the same time. The second school suggests an infective theory and the history of leukaemias of childhood frequently upholds this view. Many organisms have been incriminated but no case has yet been reproduced by the injection of any of these socalled infecting agents into the blood stream or tissues of an experimental animal. Leukaemic mothers have never been known to give birth to infants suffering from or who later have suffered from leukaemia. Occasionally, more than one member of a family suffers from the same type of leukaemia, but parents and siblings of a leukaemic patient have not been shown to be liable to develop the same blood disorder. There may be a tendency to develop blood dyscrasias in a certain family group but this is not sufficient evidence to maintain the infective theory without more laboratory proof.

\section{Clinical Classification}

Luckily, the acute leukaemias are rarely seen in childhood. Their manifestations usually arise between the ages of two and ten years. The majority ( 75 per cent.) of them belong to the small cell lymphocytic type accompanied by severe anaemia (Ramsey, 1927, and Mills, I935). About one quarter of all cases described are myeloblastic while a still smaller proportion has been made up of the monocytic cell type. Aleukaemic forms of all these disorders are relatively common in childhood and comprise about half the total number of all cases.

Leukaemias are classified according to the site of the initial hyperplasia, which is followed 
by the presence in the circulating blood and the bone marrow of an abnormal number of mature and immature leucocytes.

A. Hyperplasia of lymphoid tissue (lymphatic gland cells) produces :-

I. Lymphatic leukaemia.

2. Plasma cell leukaemia.

3. Stem cell leukaemia.

4. Aleukaemic lymphatic leukaemia.

5. Lymphosiarcoma.

B. Hyperplasia of myeloid tissue (bone marrow cells) produces :-
r. Myeloid leukaemia.
2. Eosinophil leukaemia.
3. Basophil leukaemia.
4. Megakaryocytic leukaemia.
5. Chloroma.
6. Aleukaemic myeloid leukaemia.
7. Myelosarcoma.

C. Hyperplasia of monocytic cells (reticuloendothelial system) produces :-

I. Monocytic leukaemia.

2. Aleukaemic reticuloendothelioma.

3. Reticulosarcoma.

Acute and chronic forms are seen of all these groups, the chronic type quite often becoming acute, as far as the blood picture is concerned, just before the fatal termination.

\section{Clinical Picture Common to all}

\section{Acute Types}

The course of acute leukaemia in childhood is normally short, a matter of days, weeks or less often months, before death supervenes. Apparent remissions may occur but they do not change the fatal issue. The onset may be abrupt, often with complaint of sore throat, followed by malaise and then by ulceration of the mouth and pharynx which early interferes with swallowing. Sometimes there is only indefinite evidence of decreased good health and a complaint of muscular or, less often, joint pains accompanied by a mild pyrexia.
The temperature may be high from the beginning of symptoms, continuous or remittent, changing to a swinging type with the onset of absorption from the ulcerated areas. Often, however, the temperature is not changed until late in the course of the disease. The pulse rate is raised early and may be increased by the appearance of purpuric manifestations. which almost invariably accompany the eariy or late symptoms. Gross haemorrhages may occur from any viscus, either as a precipitating symptom or later, and will materially change the pulse and respiration rates.

The respiration rate will depend on the degree of anaemia, either spontaneous or following haemorrhages and on the changed metabolic rate which inevitably accompanies the leukaemic process. The intervention of respiratory infection caused by inhalation from the upper respiratory tract must also be expected.

Cardiac complications may also arise, as a result of septic absorption as well as from myocardial degeneration due to the increasing anaemia.

The appearance of the child may be fairly normal or may be early suggestive of a severe blood disorder. $\mathrm{He}$ is nearly always well nourished, the skin being pale or waxy, especially if the purpuric tendency has caused earlier haemorrhages from the nose or gums, from the bladder or bowel. There may be sores on the lips and gums, the tongue will be furred and probably dry, ulceration. of the gums, together with swelling round the neck of the teeth and ulcerated patches on the tongue and palate may be visible at the first examination or will almost certainly appear within the first week of observation. The breath may be foetid and there may be difficulty in swallowing the saliva, so that dribbling may be an early sign. When there is early mouth infection, the cervical glands will be much enlarged and may be visible below the jaw, extending the outline of the neck to a great width. When mouth sepsis is not a prominent symptom the cervical glands are not much enlarged. This local lymphadenopathy is more commonly found in acute lymphatic leukaemia than in the same stage of acute myeloblastic leukaemia. Enlargement of 
the spleen and, less often and to a lesser degree, of the liver is found in both types although neither reaches the size found in the chronic leukaemias of an older age group.

Moderately enlarged glands may be felt and less often seen in the axillae and groins, they are usually discrete and firm in texture, they do not break down nor do they become attached to skin or deep structures. They are painless unless secondary infection invades them. Sometimes these glands are found to be present at a routine health examination, which causes the child to be referred for a blood investigation.

The size of the heart will gradually enlarge outwards, owing to dilatation, due to fatty degeneration. The heart sounds will gradually lose character, owing to the increasingly flabby nature of the myocardium. The lungs may remain clear or inhalation of toxic material may set up bronchopneumonia, which rapidly kills the patient. Haemoptysis is not common, but haematemesis more often occurs. Epistaxis is a frequent cause of trouble, as it frightens the child and is not infrequently very difficult to stop, it appears repeatedly throughout the course of the illness. Petechial haemorrhages into the skin, on the buccal mucosa, in the retina and the conjunctivae come and go throughout the short course and severe haemorrhage from the bladder may be a precipitating symptom. More often the urine may show the occasional appearance of microscopic blood or it may remain normal. Occult blood may be suggested by the offensive odour of the stools.

In those patients who linger for several months bone tenderness is common, especially of the sternum and ribs. X-ray examination then shows distortion of the periosteum by deposits, which at post mortem are found to be made up of lymphoid tissue (Baty and Vogt, 1935). In chloroma there may be pain in the head due to the myeloid infiltration of the periosteum or associated with fractures due to bone absorption. Joints are not typically affected but the pain around them may give rise to an initial diagnosis of rheumatism. The nervous system is infrequently affected. Haemorrhages may produce an upper motor neurone lesion or interfere with the integrity of the cranial nerves. Incontinence of urine and faeces often exaggerates the discomfort of the patient. Visual disturbances, due to retinal haemorrhages, are sometimes the first intimation of the disease. Death may be due to haemorrhage, intercurrent infection or heart failure.

\section{Prognosis}

This is invariably fatal within a short period of the recognized onset although prolongation of life may be artificially produced by blood transfusions. Remissions sometimes occur for no apparent reason and associated with no recognized method of treatment but they are always followed by relapse and a fatal termination.

\section{Post Mortem Findings}

Those macroscopic changes due to anaemia and haemorrhages will be found together with arcas of infiltration by lymphoid material. This lymphoid infiltration can be demonstrated in all types of tissues including the haemopoietic bone marrow which may be entirely replaced by masses of lymphocytes, myelocytes or myeloblasts. The normal red colour of the marrow is thus changed to café-au-lait or even pale grey.

\section{Treatment}

Since acute leukaemic states are invariably fatal the diagnosis must be made with certainty before informing the parents. Occasionally, spontaneous remissions or a gradual change from the acute to a chronic form may occur, so that no time period should be mentioned when discussing the future course of the disease. Because the prognosis is so poor the affected child should not be removed permanently from his home until he needs expert nursing not to be obtained at home, or until repeated haemorrhages induce the parents to ask for institutional care. The patient should be allowed to take as much exercise as he can manage but his rest periods should be long. There is no special diet to be followed but the food should be palatable, easily digested and,varied as much as possible. When the throat and mouth are 
ulcerated then ice to suck will help to reduce the swollen mucous membrane which will enable the child to swallow lukewarm or cold drinks of fruit juice and glucose, well sweetened with sugar. Ice cream and meat, fruit or milk jellies, custards and junkets, offer the most easily accepted selection of food. Whole milk should be limited to one pint daily because of its tendency to leave deposits in the gingival folds, with consequent risk of further infection, and because of its constipating effect.

Symptomatic measures to relieve pain and to prevent secondary infection are more useful than making an attempt to try drugs which cannot have any rational effect. The throat infection may be made more bearable by hourly sprays with penicillin, if the majority of the infecting organisms are shown to be penicillin sensitive. Penicillin may be given systemically in large doses, such as $\frac{1}{4}$ to $\frac{1}{2}$ million units twice daily by intramuscular injection, the treatment being restricted to the period of throat infection only. The drug has no effect on the course of the leukaemias. If many organisms of the Vincent type are grown from the throat lesions then early improvement follows the twice daily painting with $\mathrm{R} / \mathrm{gly}$ cerin, vinum ipecacuanha, liquor arsenicalis equal parts of each. This paint should be treated with care, it should be kept locked up and used only by a qualified nurse or doctor. Cold applications to the neck will often be found to be more comforting than hot fomentations, although each may find its use from day to day. The hygiene of the mouth must be strictly attended to, even in the absence of throat infection. Prophylaxis may be obtained by sucking penicillin tablets, one every hour for six hours only in any one day. This is useful if the child is old enough and well enough to co-operate, for the treatment fails in its object if the tablets do not remain in the mouth. Blood transfusions may be needed after haemorrhages which have been unduly severe or which have failed to be controlled by the ordinary haemostatic measures. However, repeated blood transfusions will keep the patient alive for a time but only for a period of increasing misery for himself and his relations. Injections of pentnucleotide may help to increase the number of adult granular leucocytes in the early days before severe damage has occurred in the haemopoietic marrow, but as the damage progresses no good result will follow such stimulants.

Folic acid has not yet been shown to have any specific effect in the leukaemias.

Nitrogen mustard (methyl-bis (B-chlorethyl) amine hydrochloride) has not proved to be useful except in a series of cases of lymphosarcoma. X-ray irradiation rarely helps acute leukaemic conditions because it accelerates the already rapid course of the disease process. It is indicated, however, when acute enlargement of the spleen causes much abdominal pain, or when enlargement of lymph nodes, as in lymphosarcoma, produces respiratory distress or difficulty in swallowing. Radium is not useful in acute leukaemias, nor have thorium$\mathrm{X}$, radium-thorium and thorium emanation provided a more hopeful outlook. Benzol, phosphorus, arsenic and antimony hold no satisfactory place in the therapeusis of this group of fatal disorders, although they may all have a useful place in the treatment of the chronic forms. Intramedullary injections of normal human bone marrow will stimulate maturation of those cells manufactured therein if introduced daily into the sternal marrow, but such treatment has obviously a very limited scope in the acute leukaemias, which rarely are seen in the very early stage.

\section{General Differential Diagnosis}

I. Acute infections producing septicaemia. Here the total number of leucocytes may be greatly increased but they will all be adult in character. The thrombocytes will not be reduced in number, as they are in the leukaemias. Blood culture should demonstrate the causative organism.

2. Acute hemolytic anaemia sometimes called acute leukaemia with recovery or acute hemolytic anaemia of Lederer. In this syndrome the red cells are gravely affected with a high proportion of immature red cells seen in each field. Blood platelets are usually normal or only slightly decreased. The moderate leucocytosis or leucopenia is usually a reaction of the myeloid cells, myelocytes and myeloblasts constantly appearing. Diagnosis may be very difficult and sternal puncture pictures may 
need to be repeated several times before their study confirms the diagnosis. Recovery is the rule with early treatment by blood transfusions and bone marrow stimulants.

3. Acholuric jaundice or hemolytic icterus. There is a history of recurrent attacks of jaundice, often in more than one member of the family. Characteristic blood findings are a high reticulocyte count, deeply staining microcytes, increased red cell fragility and a positive indirect Van den Bergh reaction.

4. Idiopathic thrombocytopenic purpura. In this condition associated with a deficiency of platelets or prothrombin the patient is not ill and shows no febrile reaction. There is no adenitis nor stomatitis and no immature white cells in the blood films. The degree of anaemia depends upon the amount of haemorrhage. The anaemia is hypochromic in character and there is no special peculiarity of the red cells. Platelets are reduced, during the active stage of the disorder, much below the normal of 400,000 to 250,000 per c.mm. Bleeding time is prolonged, but the coagulation time is normal.

5. Idiopathic aplastic anaemia. The whole function of the bone marrow has been put out of action by some as yet unrecognized defect. The anaemia is progressive and there are no remissions. There are no reticulocytes and the leucocyte count falls to 1,000 per c.mm. or even lower. No immature red or white cells are seen at any stage of the illness. Marrow puncture shows hypoplasia with a lack of mature cells, particularly affecting the granular series of leucocytes. There is often an increase of primitive cells, resembling small lymphocytes. Sometimes the marrow shows hyperplasia with a large number of primitive stem cells.

6. Von Faksch's syndrome. This condition always occurs before the age of three years. There is no enlargement of the lymph nodes unless congenital syphilis is a factor in the etiology. Anaemia may be severe but it is never profound and there is evidence of active red cell regeneration. There is a moderate leucócytosis with a small proportion of immature white cells. The platelets are not reduced and purpura and haemorrhages are not seen. The condition frequently complicates active rickets, tuberculosis, congenital syphilis and chronic gastrointestinal disturbances. Improvement takes place when a correct diagnosis is made and the appropriate specific treatment, together with that necessary for the anaemia, is established.

Bone marrow picture in children. When compared with the bone marrow of an adult that of a child shows a greater number of all types of nucleated red cells together with a higher proportion of lymphocytes and a lower proportion of myeloid cells. The total nucleated red cell count varies from 75,000 to 150,000 . The ratio of myeloid to nucleated red cells varies from 3 to I, to I to 2 .

Specimens of bone marrow for diagnosis may be made by trephine or puncture of the sternum. The latter is the more convenient in infants and children as it may be done at the bedside and repeated as often as required.

\section{A. Hyperplasia of Lymphatic Tissue}

I. Acute lymphatic leukaemia is the most common type seen in childhood. Boys are more often affected than girls, though this distinction is not so marked before six years old. Lymphadenopathy may be the presenting symptom. The cervical glands are usually more affected than those in other areas, and particularly so when the onset is acute and associated with buccal ulceration. The spleen is always enlarged. It is smooth, firm and tender, and the consequent enlargement of the abdomen may be the reason for bringing the child for medical advice. The liver is not usually greatly enlarged.

Blood changes. The red cells rapidly diminish to $\mathrm{r}, \infty 00,000$ per c.mm. and the $\mathrm{Hb}$. falls to 20 per cent. or 30 per cent. Signs of regeneration are seen in the early days but these later disappear.

The white cells are greatly increased in number, up to 100,000 per c.mm. Sudden variations may occur but have no relation to the ultimate course of the disease. Before death the total number of cells usually falls abruptly.

Lymphocytes or lymphoblasts comprise up to 99 per cent. of the total cell count. They are usually abnormal in character. The granular 
cells are relatively and absolutely reduced. Platelets are greatly reduced, so that bleeding time is prolonged and clot retraction is delayed, which causes the haemorrhagic manifestations so characteristic of the disease.

Differential diagnosis. See also general differential diagnosis.

In childhood many infectious processes produce a high lymphocytic response, so that a full examination of the blood and bone marrow biopsy pictures, together with a knowledge of the likely predisposing causes, may be required to make a correct diagnosis. In these infections, immature lymphocytes are absent and platelets are not affected.

Rubella may have a high total lymphocytic count but they are all mature cells. Plasma cells will also be found. The rash is typical.

Whooping cough shows a high count of mature lymphocytes, platelets are numerous and there is no severe anaemia.

Post-infectious lymphocytosis shows the normal or increased number of granular cells, showing toxic nuclear changes.

Septicaemia may be accompanied by a high total number of lymphocytes, which are all mature. Platelets may be diminished and the diagnosis may be difficult.

Congenital syphilis shows the enlarged glands and spleen, relative increase of mature lymphocytes and a severe anaemia. The Wassermann will be positive and the blood picture will improve with specific treatment.

Infectious mononucleosis may be distinguished by the normal number of platelets, absence of severe anaemia and frequently help may be expected from the Paul-Bunnell agglutination test.

- Essential thrombocytopenic purpura may be diagnosed by the low number of lymphocytes, most of which are mature, by the low platelet count and by the absence of lymphadenopathy.

Scurvy should be differentiated by the normal number of mature lymphocytes and platelets.

Agranulocytic angina is occasionally very difficult to differentiate. The lymphocytes are mature and the granular series of leucocytes rapidly disappear.

Acute rheumatism may be differentiated by the blood picture, which may be aleukaemic, but all the cells are mature and the normal balance of the differential count is maintained.

Treatment. There is no specific treatment. Blood transfusions may prolong life.

Case History. Lymphatic leukaemia. Sylvia aged four years.

One month before admission on 9.6.43, the nurse at a L.C.C. Cleansing Station had observed a swelling in the left axilla, while the child was under treatment for scabies. She was referred to hospital. She was reported to have a poor appetite and a slight non-productive cough, otherwise her health was stated to be as usual. She had had measles and chicken pox.

There were eight other children in the family, all well. The father was alive and well, the mother had arthritis.

On examination: Well built and well covered. Skin yellowish in colour-mucous membranes pale. Glands enlarged, hard and discrete in neck, axillae and inguinal regions. No tenderness and no skin attachment. Spleen palpable three fingers below the costal margin. Liver palpable two fingers below the costal margin. Throat clean and no signs in chest to account for cough.

\section{Blood Counts}

$$
\begin{aligned}
& \text { Hb. R.B.C. W.B.C. } \\
& \text { 8.6.43 79\% } \quad 4,005,000 \quad 37,000 \\
& \begin{array}{llll}
\text { Myelocytes } & \ldots & \ldots & \text { I }
\end{array} \\
& \text { L.Lymphs } \quad \ldots \quad \ldots \quad 38 \\
& \text { S.Lymphs } \ldots \quad \ldots \quad \ldots 45 \\
& \begin{array}{lllll}
\text { Smudges } & \ldots & \ldots & \ldots & \mathbf{6 . 5}
\end{array} \\
& \text { Lymphoblasts _. } \quad \ldots \quad \text { I } 3 \\
& \text { Polymorphs } \quad \ldots \quad \ldots \quad \text { I } \\
& \begin{array}{ccccc}
\mathbf{1 4 . 6 . 4 3} \begin{array}{c}
\text { Hb. } \\
66 \%
\end{array} & \begin{array}{c}
\text { R.B.C. } \\
3,335,000
\end{array} & \multicolumn{2}{c}{\begin{array}{c}
\text { W.B.C. } \\
33,800 \\
\%
\end{array}} \\
\text { Myelocytes } & \ldots & \ldots & 0.5 \\
\text { Myeloblast . . } & \ldots & \ldots & 0.5 \\
\text { Lymphocytes } & \ldots & \ldots & 81.5 \\
\text { Lymphoblast } & \ldots & \ldots & 5 \\
\text { Smudges .. } & \ldots & \ldots & 12 \\
\text { Polymorphs } & \ldots & \ldots & 0.5
\end{array}
\end{aligned}
$$


Most of the 8I per cent. lymphocytes are abnormal in structure and may be classed as abnormal 1ymphoblasts.

$$
\begin{aligned}
& \text { Hb. R.B.C. W.B.C. } \\
& \text { 22.6.43 } 63 \% \quad \text { - } \quad 14,100 \\
& \begin{array}{llll} 
& & & \% \\
\text { Myelocytes } & \ldots & \ldots & 0.5
\end{array} \\
& \text { Normal lymphocytes } \quad \ldots \quad 36.5 \\
& \text { Abnormal lymphocytes ... } 17.5 \\
& \text { Lymphoblasts . . } \quad \ldots \quad 6 \\
& \begin{array}{llll}
\text { Monocytes .. } & \text {. } & \text {. } & \text { I.5 }
\end{array} \\
& \begin{array}{llll}
\text { Smudge cells } & \ldots & \ldots & 37.5
\end{array} \\
& \begin{array}{llll}
\text { Polymorphs } & \ldots & \ldots & 0.5
\end{array} \\
& \text { Hb. R.B.C. W.B.C. } \\
& \text { 30.6.43 } 3 \mathrm{r} \% \quad-33,600 \\
& \begin{array}{llll}
\text { Myelocytes } & \ldots & \ldots & \% \\
& 0.5
\end{array} \\
& \text { Lymphocytes } \quad \ldots \quad \ldots \quad 34.5 \\
& \text { Lymphoblasts . . . } 65.0
\end{aligned}
$$

Very few platelets seen. Bone marrow from sternal puncture. Marked infiltration with mononuclear cells resembling those in the blood stream. No granular cells seen.

Treatment: I. Fe and Amm. Cit. gr. xv t.d.s. started on day of admission.

2. Liq. arsenicalis m. ii Aq. Ch. $\mathrm{Cl}_{3}$ ad. 3 fs. daily.

3. Ascorbic acid $100 \mathrm{mg}$. b.d.

4. Nicotinic acid $50 \mathrm{mg}$. b.d. all started on day of admission.

5. Blood transfusions given on two occasions (3 oz. each).

6. Vitamin $\mathrm{K}$ given by the mouth, ro $\mathrm{mg}$. daily.

7. Sedatives beginning with chloral and ending with morphia.

Progress. Afebrile, except for occasional rise to $99^{\circ}$ until seven days before death on 4.7 .43 , i.e., 26 days after admission. Small ulcer appeared on upper gum and one attack of epistaxis, followed by petechial haemorrhage into tonsillar region. No other haemorrhages throughout. No post-mortem findings available.

2. Plasma cell leukaemia resembles lymphatic leukaemia except that the predominant cell in the blood picture is the plasma cell, which is a normal product of the red bone marrow. Infiltration of plasma cells may occur into the eye tissues, the liver, spleen, bone marrow and bones, and the upper respriatory passages. Differential diagnosis from multiple myelomatosis may be very difficult.

3. Stem cell leukaemia is the term given when staining fails to differentiate one group of predominant cells from another type. The stem cell is the precursor of myeloid and lymphoid cells and may increase in number without any differentiation in an acute leukaemic process. The clinical symptoms resemble those of acute lymphatic leukaemia.

4. Lymphosarcoma. This is a primary malignant disease of lymphatic tissue developing from atypical lymphocytes in lymph nodes or from areas of lymphoid deposits in any tissue. The glands most frequently affected are those of the neck, the mediastinum, the spleen and Peyer's patches in the intestines. The neoplasm is locally malignant and gives rise to destruction of tissues adjacent to the primary growth by direct infiltration and to metastases by lymphatic spread. Occasionally a leukaemia picture, both clinical and blood, supervenes on the more normal course of lymphosarcoma. The course is always short with a fatal termination. Treatment until recently consisted of early heavy irradiation of the affected glands, together with the use of arsenicals. Now, the use of methyl-bis (B-chloroethyl) amine hydrochloride (nitrogen mustard) in a series of cases has produced hopeful evidence that immediate alleviation of symptoms, both local and general, is available to patients. The time during which the drug has been tested has been too short to give an indication of the ultimate life-span of those who receive it at a sufficiently early stage of their disease, (Jacobson et al., F.A.M.A., I32, No. 5, p.263).

\section{B. Hyperplasia of Myeloid Tissue}

I. Acute myeloid leukaemia (myeloblastic leukaemia, splenomedullary leukaemia, myelosis). The clinical symptoms and blood films often so resemble those found in acute lymphatic leukaemia that a precise diagnosis is difficult. The onset is usually abrupt with sore throat and a raised temperature. The course is usually very short. The fatal termination is unaffected by present day treatment. The condition frequently terminates the chronic type of myeloid leukaemia. The mouth 
and pharyngeal sepsis is early and severe, the local appearance being suggestive of acute agranulocytopenic angina or diphtheria. Glands are not greatly enlarged except those related to pharyngeal sepsis. The spleen is usually moderately enlarged, together with some degree of hypertrophy of the liver. Anaemia, due to haemorrhages, which occur as the platelet count falls, is progressive and becomes more marked as the red bone marrow becomes replaced by leukaemic infiltration.

Blood changes. The red cells rapidly fall to I,000,000 per c.mm. or less. The haemoglobin also falls early so that the colour index remains about $I$. Signs of regeneration, shown by nucleated red cells, reticulocytes, and all forms of immature red cells are seen at first but these later disappear permanently. The white cell count is greatly increased, up to 100,000 per c.mm. Variations in number occur from day to day and bear no relation to the ultimate fatal result. The majority of the cells seen in a blood film are myeloblasts and myelocytes, which at first look like large lymphocytes. Distinction is made by a positive oxidase test.

The mature granular leucocytes are absolutely and relatively reduced so that the immature cells constitute about 50 per cent. to 90 per cent. of. the total cell count. The blood platelets decrease progressively as the bone marrow is invaded by the leukaemic material, so that bleeding time and clot retraction time are increased. Haemorrhages are a direct sequel to this lowered platelet count.

Differential diagnosis. As in acute lymphatic leukaemia, acute infections of the specific types and those associated with blood infections may be differentiated by a careful estimation of the type of leucocyte seen in $b^{\prime}$ ood films and by the presence of normal numbers of platelets. Blood culture may be required. Scurvy, agranulocytopenic purpura and aplastic anaemia may be differentiated by the absence of immature leucocytes in the blood and bone marrow, and the other characteristics of each separate disorder.

\section{Acute eosinophil}

3. Acute basophil and

4. Acute megakaryocytic leukaemia may be diagnosed occasionally by the presence of a leucocytosis, made up principally of eosinophils, basophils or platelets. Such blood pictures are usually superimposed on that of a chronic myeloid leukaemia. In this disease the prognosis is made worse by the appearance of basophil cells in large numbers in the blood picture. The megakaryocytic type is found with great enlargement of the spleen and a diagnosis of splenic anaemia is made at first. Treatment is unavailing. Fatal results always occur.

5. Chloroma. The characteristic sign of this type of myeloid neoplasm is the invasion of the tissues by greenish myeloblastic tumours. The bones of the skull and thorax are most commonly affected. The green colour of the cells disappears on exposure to light. Most commonly seen in children, the disease usually has a fulminating course, unaffected by treatment, and death follows in a few weeks from the onset. The clinical symptoms are those common to all the acute leukaemias. The face changes its shape because of the deposits in the orbits and facial paralysis may result. Exophthalmos is common, blindness is caused by the retrobulbar pressure and greenish deposits may be seen in the sclerae, while the cornea becomes cloudy and ulcerated. The ears are frequently invaded, so that deafness results. Epistaxis and later nasal obstruction follows involvement of the nasal bones. Petechial haemorrhages occur in the skin and elsewhere and there may be profuse bleeding from any múcous surface.

$\mathrm{X}$-ray changes in the affected bones are those of decalcification and absence of regeneration. The skull, vertebrae and bones of the pelvis show the most characteristic changes.

Blood picture. There is a severe anaemia of the hypochromic type. Signs of active regeneration are usually found though there may be an aplastic picture. The leucocytes are moderately increased and their appearance is difficult to distinguish from an acute myeloid leukaemia. The immature white cells are always myeloblasts. This blood picture is the method of differentiation from scurvy, xanthomatosis, neuroblastoma of the adrenals and sarcoma. 
6. Aleukaemic myeloid leukaemia frequently occurs as a variant of acute myeloid leukaemia. Its clinical course is similar.

Case report. Aleukaemic myeloid leukaemia. Hazel aged 8 years.

For two months before admission on 30.10.42, child had been easily tired and listless. She had occasionally complained of pain in the shoulders and of headache. She was referred by the school doctor, who noted her pale appearancè during a routine school examination. She had had measles, rubella, pertussis, mumps and chicken pox. She had one sister, aged 14 years, who was alive and well. Parents both alive and well.

On examination : Plump, well grown child. Skin very pale, as were mucous membranes. Bruise on right arm and on right thigh. Fauces slightly injected. Two small discrete cervical glands felt, no other glandular enlargement. Liver and spleen not enlarged.

\section{Blood Counts}

$$
\begin{aligned}
& \text { Hb. R.B.C. W.B.C. } \\
& \text { 30.10.42 } 41 \% \quad \text { I }, 576,000 \quad 3,650 \\
& \begin{array}{lllr}
\text { Myeloblasts } & \ldots & . & \% \\
2
\end{array} \\
& \text { Premyelocytes } \quad \ldots \quad \ldots \quad \text { I } \\
& \begin{array}{lllll}
\text { Myelocytes } & \ldots & \ldots & \ldots & 5
\end{array} \\
& \text { Stab and band forms } \quad \ldots \quad 17 \\
& \text { Polymorphs } \quad \text {. } \quad \ldots \quad 9 \\
& \text { Lymphocytes } \quad \ldots \quad \ldots 43 \\
& \text { Monocytes .. } \quad \ldots \quad \ldots \quad 3 \\
& \text { Hb. R.B.C. W.B.C. } \\
& 31.10 .42 \quad 38 \% \quad \mathrm{I}, 106,000 \quad 3,320
\end{aligned}
$$

Red cells large and well filled.

$$
\begin{aligned}
& \begin{array}{rrrr} 
& \text { Hb. . } & \text { R.B.C. } & \text { W.B.C. } \\
16.11 .42 & 28 \% & 1,005,000 & 2,670
\end{array} \\
& \begin{array}{llll}
\text { Myeloblasts } & \ldots & \ldots & \% \\
& \text { I I }
\end{array} \\
& \begin{array}{lllr}
\text { Premyelocytes } & \ldots & \ldots & 11 \\
& & \ldots & 6
\end{array} \\
& \begin{array}{llll}
\text { Myelocytes .. } & \ldots & \ldots & 16
\end{array} \\
& \text { Abnormal forms } \quad \ldots \quad \ldots \quad 27 \\
& \text { Polymorphs } \quad \ldots \quad \ldots 2 \text { 2I } \\
& \text { Lymphocytes } \quad \ldots \quad \text {. } 10 \\
& \text { Monocytes .. } \quad \ldots \quad \ldots \quad 9
\end{aligned}
$$

Bone marrow from sternal puncture. Platelets few and very large. Differential count showed varying types of myeloblasts, many being vacuolated. Numerous more immature blasts of the haemocytoblast type present. Picture of acute myeloblastic leukaemia.

Treatment. Fersolate, gr. iii t.d.s.

Benerva, mgm. iii t.d.s.

Ascorbic acid, mgm. I 50 daily.

Ventriculin, one capsule daily given from day of admission.

Progress. Child sent home four days after admission, but had to be re-admitted on I6. I 1.42 because her mother became ill. On the following day, epistaxis was followed by a large haematemesis, which was repeated several hours later. Child became comatose and died within 12 hours.'

Post-mortem findings. Petechial haemorrhages on surface of lungs, heart and mucous surface of stomach. Right heart dilated, muscle pale and flabby. No blood in stomach or gut. Liver not enlarged but pale and fatty. Spleen not enlarged. Kidneys pale and fatty. Bone marrow of femur red throughout.

\section{Hyperplasia of Monocytic Tissue}

I. Acute monocytic leukaemia. The diagnosis of this type of leukaemia is always difficult, for it depends upon the recognition of the monocytes which make up the predominating proportion of leucocytes in the blood picture. Monocytes of all degrees of maturity may occur and as the very primitive types are similar to myeloblasts, and myelocytes and lymphoblasts are forced into the blood stream by monocytic invasion of the bone marrow, the blood picture may be very confusing. Bone marrow biopsy is the most important method of making a diagnosis, for monocytes are not normally seen in a marrow film.

In the variant of monocytic leukaemia where myelocytes and monocytes are seen in large numbers, it is thought by some haematologists that the condition is a sub-group of myeloblastic leukaemia. This type is called the Naegeli group. Many observers, however, maintain that the monocytes are derived from the reticuloendothelial system and call it the Schilling type. Clinical features are similar to those already described. Ulceration of the mouth and throat is almost always seen, and enlargement of the cervical glands due to this 
infection is the rule. The liver is moderately enlarged but the spleen rarely enlarges appreciably. The skin is covered in a macular rash, due to infiltration with monocytic deposits, and petechial haemorrhages frequently erupt in crops at different times during the illness.

Blood picture. The red cells and haemoglobin fall progressively, giving a picture of hypochromic anaemia. Marked signs of regeneration are always seen. Platelets are reduced, with decreased clot retraction and prolonged bleeding time. The leucocytes are moderately increased up to 50,000 per c.mm. with a rapid rise just before death. Fluctuation in number is marked. The differential count shows that 50 per cent. -90 per cent. of the leucocytes are monocytes.

Differential diagnosis must be made from septicaemia, Vincent's angina, glandular fever and lymphatic leukaemia. Prognosis is always bad; remissions, which frequently appear, having no effect on the ultimate fatal result. Treatment is only usefully employed for symptomatic relief.

2. Leukoreticulo-endothelioma. Reticulosarcoma. This type of neoplasm arises in the cells of the reticuloendothelial system and is always associated with a monocytic leukaemic blood picture. Deposits of neoplastic material occur in the spleen, liver, lymph nodes and metastases occur in the skin, mediastinum and abdomen. Treatment is again only symptomatic.

Whoever is to acquire a competent knowledge of medicine, ought to be possessed of the following advantages : a natural disposition; instruction; a favourable position for the study; early tuition; love of labour; leisure. First of all, a natural talent is required; for, when Nature leads the way to what is most excellent, instruction in the art takes place, which the student must try to appropriate to himself by reflection, becoming an early pupil in a place well adapted for instruction. He must also bring to the task a love of labour and perseverance, so that the instruction taking root may bring forth proper and abundant fruits.

THE LAW OF HIPPOCRATES. 